\title{
Humanitarian relief supply chain: a multi-objective model and solution
}

\author{
ADITYA JHA ${ }^{1}$, DAMODAR ACHARYA ${ }^{2}$ and M K TIWARI ${ }^{2, *}$ \\ ${ }^{1}$ Department of Mechanical Engineering, Indian Institute of Technology Kharagpur, Kharagpur 721302, India \\ ${ }^{2}$ Department of Industrial and Systems Engineering, Indian Institute of Technology Kharagpur, Kharagpur \\ 721302, India \\ e-mail: adityajha36@gmail.com; acharyadamodar94@gmail.com; mkt09@hotmail.com
}

MS received 19 November 2016; accepted 26 May 2017

\begin{abstract}
This paper models a humanitarian relief chain that includes a relief goods supply chain and an evacuation chain in case of a natural disaster. Optimum network flow is studied for both the chains by considering three conflicting objectives, namely demand satisfaction in relief chain, demand satisfaction in evacuation chain and overall logistics cost.The relief goods supply chain consists of three echelons: suppliers, relief camps and affected areas. The evacuation chain consists of two echelons: evacuation camps and affected areas. The model has been made more resilient by considering multiple paths between any two locations and disruption of camps and paths due to natural factors. The Mixed Integer Programming problem has been solved using NSGA-III and results have been compared to those from benchmark algorithms. The model has been successfully tested on generated real-life-like data.
\end{abstract}

Keywords. Humanitarian logistics; supply chain resilience; multiple-objective programming; evolutionary algorithms.

\section{Introduction}

According to the Indian National Disaster Management guidelines [1], floods occur very frequently in various parts of the country and cause huge loss of life and damage to public utilities and infrastructure. In India, around 40 million hectares out of a total land area of 329 million hectares is prone to floods. In flooded areas every year around 1600 lives are lost, 7.5 million hectares of land is affected and damage is caused to public facilities, houses and crops, amounting to $\$ 285$ million [2].

In the event of a flood a decision maker is faced with problems of conflicting objectives and insufficient data. Thus, it is important that any model concerning these issues handles both of these important characteristics, namely conflicting objectives and insufficient data. Construction of suitable shelters for the victims is an essential component in the preparedness for floods during the relief period. Such shelters are designed by the state government, keeping in mind the area's climatic conditions and the functional needs of the people. Appropriate locations for the construction of such shelters are identified to ensure that the minimum needs for hygiene are satisfied.

The objective of the response during a disaster is to provide relief goods (like water, food, medicine, etc.) and

*For correspondence evacuate as many people as possible from the affected areas (AAs) in minimal time so as to minimize human suffering and death. To minimize the time, large scale suppliers like private manufacturing units as well as government storehouses send their goods to the relief camps, established near the AAs. From the relief camps the goods are sent via various paths to the AAs. The evacuation camps are often set up on the outskirts of the district closest to the AAs, so that they can get their supplies easily. It helps in easy maintenance and is also helpful while providing the victims with regular medical check-ups.

This study offers decision makers with a very wide range of possibilities to deal with the disaster scenarios and provides a model into which many other objectives can be included, to make it more practical, in offering a solution to a multi-objective problem. The problem is solved by a genetic algorithm that can handle up to 15 objectives. Hence, there is a lot of scope for improving the number of objectives in the problem. Genetic algorithms are very popular among supply chain researchers owing to their ability to evolve, tolerate uncertainty and execute optimization by robust simulations of human decision making behaviour [3, 4].

The rest of the paper is organized as follows. A thorough literature review of previous work done in relief logistics is discussed in section 2. The complete problem is described in section 3, while section 4 presents the mathematical 
formulation of the model. Section 5 contains the complete description of multi-objective optimization and NSGA-III. Section 6 presents the results and observations when the problem is solved using NSGA-III and MOEA/D approach. The conclusion and scope for the future work is given in section 7 .

\section{Literature review}

In this section, we review the literature on disaster relief logistics. Despite the availability of broad literature reviews on disaster relief and humanitarian aid, specific areas like relief goods distribution of humanitarian logistics [5, 6] have had limited research. A number of articles giving an overview on humanitarian logistics [5, 7] and relief distribution networks are also available, with a few of them focussing specifically on the case of India [8, 9]. Facility location, network flow problems and inventory management are three basic streams into which the related literature in the context of disaster relief logistics falls.

Toregas et al [10] were among the first researchers to analyse the optimal locations of the emergency facilities. Emergency response plans were developed by Parentela and Nambiran [11]. Akkihal [12] stressed the optimization of possible locations for the storage of non-consumable aids. Balcik and Beamon [13] used a fuzzy multi-objective programming method for optimizing the demand of the relief chain and the number and location of relief distribution centres. Tzeng et al [14] considered the cost, demand satisfaction, time to respond and optimal distribution of commodities to demand points in their multi-objective deterministic model. Ukkusuri and Yushimito [15] considered the disruption of the transport network and thus developed a model to maximize the probability to reach the demand points from a single supply facility. A study of optimization models in the context of emergency logistics can be found in [16] and [17].

Research on inventory management focusses on deciding the amount of relief goods demanded by different relief camps along the chain and the safety stock amount to be maintained is also determined. Whybark [18] emphasized disaster inventories and stressed the importance of the gathering, storage and distribution of products. Ozbay and Ozguven [19] developed a model of inventory control for safety stock levels that was time dependent. Beamon and Kotleba [20] determined optimal order quantities and reordered pre-positioned warehouse points during a long-term emergency relief response. Rachaniotis et al [21] discussed multiple location and routing models in humanitarian logistics.

Network flow is classified into two broad classes: relief distribution and victim evacuation. [22-25] and [26] have all done research work on victim evacuation. Zhou et al [27] have combined fuzzy logic and a decision making trial and evaluation laboratory (DEMATEL) to determine the critical success factors of emergency management.
Ben-Tal et al [28] applied robust optimization (RO) and dynamically assigned emergency response with time-dependent demand uncertainty. A survey of operations research models was presented by [29] to tackle vehicle routing problems in the disaster phase. [30, 31] and [32] have proposed models for relief distribution. Hadiguna et al [33] have designed a web-based decision support system to assess the feasibility of public facilities during evacuation.

The use of stochastic optimization in disaster relief preparedness, which involves the distribution of relief supplies under probabilistic scenarios representing a disaster, has a significant level of uncertainty. Research addressing such disaster relief planning involves mostly models of stochastic situations under demand uncertainty or under demand and supply uncertainty.

\section{Problem description}

In this model, we assume two chains, a supply chain and an evacuation chain, having three and two stages, respectively. In the supply chain, the first stage is the suppliers, like the private and government agencies. The second stage is the set of relief camps, where the emergency supply goods will be stored. The third stage consists of the areas affected by the disaster (AAs). In the evacuation chain, the first stage is the evacuation camps, which act as temporary residences for the people rescued from the AAs. The second stage consists of the areas affected by the disaster (AAs). Multiple paths are considered between each relief camp/evacuation camp and the AA. They can also represent the different modes of transportation. This makes the model more robust.

Concerning the selection of the location of the relief and evacuation camps from a group of candidate locations, certain issues that need to be addressed include the capacity of the camp at a particular location and the distance to the AAs that keeps the transportation cost to a minimum. An issue of practical importance, the disruption probability of the relief and evacuation camps due to the destruction, is also considered. Another issue considered is the risk associated with a particular path, since a decision maker does not have control over factors such as the damage of transportation routes in such scenarios. Such a damage will affect the cost of transportation along that path. Various scenarios with different probabilities have been considered. The damage to the path and camps will be different in different scenarios. Figure 1 gives a general representation of our model.

We have made certain assumptions:

1. The capability of suppliers is not affected by a disaster or any disruption to the roads and facilities.

2. Instead of including multiple commodities in our model, we have considered a single package, which consists of different commodities such as food, water or medicine. 


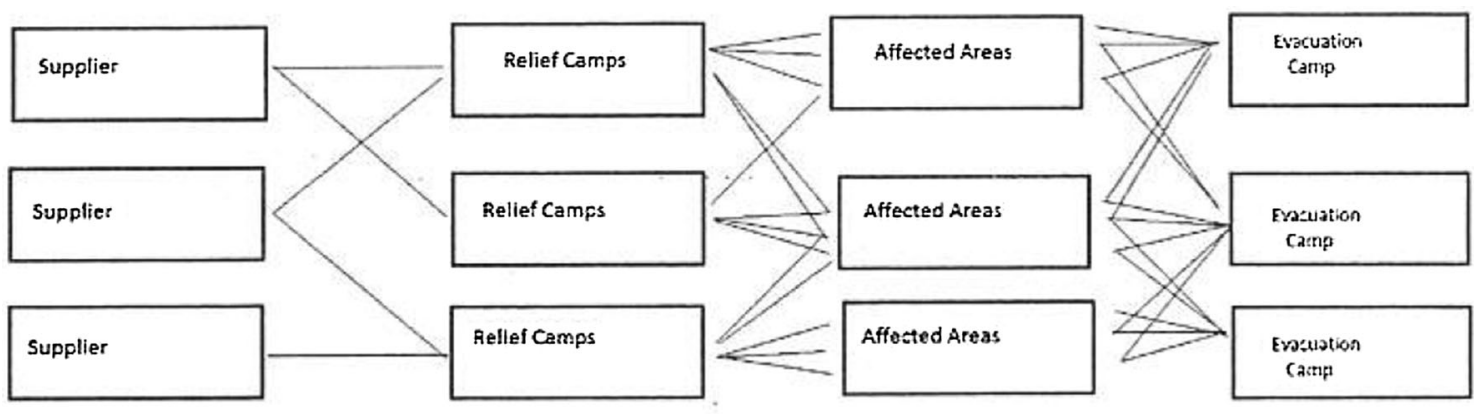

Supply Chain

Evacuation Chain

Figure 1. A representation of the proposed model.

3. A relief camp or an evacuation camp can be opened with only one of the three possible configurations (small, medium or large). The sizes of the small, medium and large camps are taken in the ratio of 1:2:3. The cost of opening a relief camp/evacuation camp is directly proportional to the size of the camp, the proportionality constant being different for each relief camp.

4. Each AA can be served by multiple relief and evacuation camps.

5. Only one path is selected for transportation between a particular relief camp/evacuation camp and an AA.

\section{Mathematical formulation}

The following notations given in tables 1, 2 and 3 are used to formulate the supply and evacuation pre-positioning model.

\subsection{Components of objectives}

$S C_{j} \quad$ (set-up cost of relief camp at $j$ ) $=\sum_{j=1}^{J} C_{j} Z_{j}$

$S C_{h} \quad$ (set-up cost of evacuation camp at $h$ ) $=\sum_{h=1}^{H} C_{h} Z_{h}$

PC (procurement cost from supplier $i$ )

$$
=\sum_{i=1}^{I} \sum_{j=1}^{J} s c_{i} \times X_{i j}
$$

Table 1. Sets/indices.

\begin{tabular}{|c|c|c|c|}
\hline$I$ & $\begin{array}{c}\text { Set of suppliers indexed } \\
\text { by } i \in I\end{array}$ & $J$ & $\begin{array}{c}\text { Set of relief camps indexed } \\
\text { by } j \in J\end{array}$ \\
\hline$K$ & $\begin{array}{l}\text { Set of affected areas } \\
\text { (AAs) indexed by } \\
\qquad k \in K\end{array}$ & $M$ & $\begin{array}{l}\text { Possible paths between a } \\
\text { relief camp and an AA }\end{array}$ \\
\hline$S$ & $\begin{array}{c}\text { Set of possible scenarios } \\
\text { indexed by } s \in S\end{array}$ & $P$ & $\begin{array}{c}\text { Paths between an } \\
\text { evacuation camp and an } \\
\text { AA }\end{array}$ \\
\hline$H$ & $\begin{array}{l}\text { Set of evacuation camps } \\
\text { indexed by } h \in H\end{array}$ & & \\
\hline
\end{tabular}

SC (stay cost at evacuation camp $h$ ) $=\sum_{h=1}^{H} \sum_{k=1}^{K} \sum_{p=1}^{P} N_{h k p} S_{h}$

TCS (transportation cost from suppliers) $=\sum_{i=1}^{I} \sum_{j=1}^{J} c_{i j} X_{i j}$

$D R_{s c} \quad$ (disruption penalty cost for supply chain)

$$
=K_{1} \sum_{j=1}^{J} \sum_{s=1}^{S} p_{s} \times d i s_{j s} \times Z_{j}
$$

Table 2. Parameters.

$b \quad$ Portion of area occupied by one product at a small relief camp

$c_{i j} \quad$ Transportation cost of a unit product from supplier $(i)$ to relief camp $(j)$

a Portion of area occupied by one person at a small evacuation camp

$K_{1} \quad$ A constant to normalize disruption probability into cost

$K_{2} \quad$ A constant to normalize risk associated with path into cost

dis $_{j s} \quad$ Disruption probability of relief camp at $j$ in scenario $s$

$d i s_{h s} \quad$ Disruption probability of evacuation camp at $h$ in scenario $s$

$r_{j k m} \quad$ Path risk associated with the path $m$ between relief camp $j$ and affected area $k$

$r_{h k p} \quad$ Path risk associated with the path $p$ between evacuation camp $h$ and affected area $k$

$c_{j k m} \quad$ Transportation cost of unit item from relief camp $j$ to affected area $k$ via path $m$

$c_{h k p} \quad$ Transportation cost of unit item from evacuation camp $h$ to affected area $k$ via path $p$

$S C_{i} \quad$ Supplier capacity of $i^{\text {th }} \quad p_{k} \quad$ Demand of relief Supplier goods at $k^{\text {th }}$ Affected area

$E_{k} \quad$ Demand of evacuation of $\quad \mathrm{Cap}_{j}$ people from $k^{\text {th }}$ affected Capacity of small relief camp $(j)$ area

$\mathrm{Cap}_{h}$ Capacity of small evacuation camp \& $h$

$c_{h} \quad$ Cost factor for setting up $p_{s}$ an evacuation camp $(h)$

$c_{j} \quad$ Cost factor for setting up a relief camp $(j)$ Probability of occurrence of scenario $s$

$s c_{i} \quad$ Procurement cost of unit $\quad S_{h} \quad$ Stay cost of one item from supplier at $i \quad$ person at evacuation camp $h$ 
Table 3. Decision variables.

\begin{tabular}{|c|c|c|}
\hline$X_{i j}$ & \multicolumn{2}{|c|}{ Number of units transported from supplier $i$ to relief camp $j$} \\
\hline$Y_{j k m}$ & Number of units trans & oorted from relief camp $j$ to affected area $k$ via path $m$ \\
\hline$N_{h k p}$ & Number of units transpor & ted from evacuation camp $h$ to affected area $k$ via path $p$ \\
\hline$Z_{j}($ area of relief camp) & $\left\{\begin{array}{l}0 \\
b \times C a p_{j} \\
2 b \times C a p_{j} \\
3 b \times C a p_{j}\end{array}\right.$ & $\begin{array}{l}\text { If there is no relief camp at } j \\
\text { If there is a relief camp of small size at } j \\
\text { If there is a relief camp of medium size at } j \\
\text { If there is a relief camp of large size at } j\end{array}$ \\
\hline$Z_{h}($ area of evacuation camp $)$ & $\left\{\begin{array}{l}0 \\
a \times \mathrm{Cap}_{h} \\
2 a \times \mathrm{Cap}_{h} \\
3 a \times \mathrm{Cap}_{h}\end{array}\right.$ & $\begin{array}{l}\text { If there is no evacuation camp at } h \\
\text { If there is an evacuation camp of small size at } h \\
\text { If there is an evacuation camp of medium size at } h \\
\text { If there is an evacuation camp of large size at } h\end{array}$ \\
\hline$\lambda_{j k m}$ & $\begin{cases}1 & \text { If path } \\
0 & \text { Otherw }\end{cases}$ & $\begin{array}{l}m \text { is selected between relief camp }(j) \text { and AA }(k) \\
\text { ise }\end{array}$ \\
\hline$\lambda_{h k p}$ & $\begin{array}{l}\text { If path } p \text { i } \\
\text { Otherwis }\end{array}$ & s selected between evacuation camp $(j)$ and AA $(k)$ \\
\hline
\end{tabular}

$D R_{e c} \quad$ (disruption penalty cost for evacuation chain)

$$
=K_{1} \sum_{h=1}^{H} \sum_{s=1}^{S} p_{s} \times d i s_{h s} \times Z_{h}
$$

TCR (transport cost from relief camps)

$=\sum_{j=1}^{J} \sum_{k=1}^{K} \sum_{m=1}^{M} Y_{j k m} c_{j k m}$

TCE (transportation cost to evacuation camp)

$=\sum_{h=1}^{H} \sum_{k=1}^{K} \sum_{p=1}^{P} N_{h k p} c_{h k p}$

Risk $_{s c} \quad$ (risk penalty for path risks in supply chain)

$=\sum_{j=1}^{J} \sum_{k=1}^{K} \sum_{m=1}^{M} \lambda_{j k m} r_{j k m}$

Risk $_{\text {ec }} \quad$ (risk penalty for path risks in evacuation chain)

$=\sum_{h=1}^{H} \sum_{k=1}^{K} \sum_{p=1}^{P} \lambda_{h k p} r_{h k p}$

$C S_{s c} \quad$ (measure of customer satisfaction for supply

chain $)=\sum_{k=1}^{K}\left(\sum_{j=1}^{J} \sum_{m=1}^{M} Y_{j k m}\right)-p_{k}$

$C S_{e c} \quad$ (measure of customer satisfaction for evacuation chain $)=\sum_{k=1}^{K}\left(\sum_{h=1}^{H} \sum_{p=1}^{P} N_{h k p}\right)-E_{k}$

Min obj1 $=S C_{j}+S C_{h}+P C+S C+T C S+D R_{s c}$ $+D R_{e c}+T C R+T C E+R_{i s k_{e c}}+$ Risk $_{s c}$

Min obj2 $=C S_{s c}$

Min obj3 $=C S_{e c}$

subject to the constraints:

$$
\begin{gathered}
\sum_{j=1}^{J} X_{i j} \leq S C_{i} \quad \forall i=1,2,3, \ldots, I \\
b \times \sum_{j=1}^{J} X_{i j} \leq Z_{j} \quad \forall j=1,2,3, \ldots, J \\
\sum_{k=1}^{K} \sum_{m=1}^{M} Y_{j k m} \leq \sum_{i=1}^{I} X_{i j} \quad \forall j=1,2,3, \ldots, J \\
\sum_{j=1}^{J} \sum_{m=1}^{M} Y_{j k m} \leq p_{k} \quad \forall k=1,2, \ldots, K \\
\sum_{m=1}^{M} \lambda_{j k m}=Z_{j} \quad \forall j, k
\end{gathered}
$$

$$
\begin{gathered}
Y_{j k m} \leq M \lambda_{j k m} \\
a \times \sum_{k=1}^{K} \sum_{p=1}^{P} N_{h k p} \leq Z_{h} \quad \forall h=1,2,3, \ldots, H \\
\sum_{n=1}^{N} \sum_{h=1}^{H} N_{h k p} \leq E_{k} \quad \forall k \\
\sum_{m=1}^{M} \lambda_{h k p}=Z_{h} \quad \forall h, k \\
N_{h k p} \leq M \lambda_{h k p} .
\end{gathered}
$$

The objectives are to minimize the total and to maximize the customer satisfactions in the relief chain and the evacuation chain. Constraint (1) ensures that the total supply from a supplier must be less than or equal to its capacity. Constraint (2) is to restrict the amount of relief goods being received by a relief camp to its maximum capacity. The number of units leaving the relief camps is less than or equal to the amount being received is ensured by constraint (3). Constraint (4) is the demand constraint, which ensures that the supply at an AA should be less than the demand to avoid wastage of resources, while constraint (5) ensures that paths are selected only from locations having relief camps. In constraint (6), $M$ is a very large number and ensures no supply via paths that are not selected. Constraint (7) ensures that the area occupied by victims at an evacuation camp is less than or equal to the area of the evacuation camp. Constraint (8) is the demand constraint, which ensures that the number of people being evacuated is less than or equal to the demand, while constraint (9) ensures that paths are selected only from locations having evacuation camps. In the final constraint, $M$ is a very large number and ensures no evacuation via paths not selected.

The model has been solved using a multi-objective optimization approach using NSGA-III. 


\section{Non-dominated sorting genetic algorithm-III (NSGA-III)}

NSGA III, formulated by Jain and Deb [34], is an elitist non-dominated sorting genetic algorithm. It is used to solve multi-objective optimization problems. NSGA III converges to the global Pareto-optimal front while maintaining the diversity of the population on the Pareto-optimal front.

The main concept of this algorithm lies in the nondomination between two solutions. If, for an $n$-objective problem, one solution is better than another in all objectives, then it is said to dominate that solution; if it is worse in all, then it is said to be dominated. In all other cases, they are non-dominating.

For a constrained optimization problem, a solution $p$ will dominate a solution $q$ if (a) $q$ is infeasible and $p$ is a feasible solution, (b) both solutions $p$ and $q$ are infeasible, but the constraint violation of $p$ is less than that of $q$ and (c) both solutions $p$ and $q$ are feasible, and solution $p$ dominates solution $q$.

The steps involved in the NSGA-III algorithm are described below:

1. Initialize the population. The initial population (of size $N$ ) is generated as per the constraints using uniformly distributed random numbers. This initial set of solutions is the first parent solution and is stored in set $P_{t}$

2. Crossover and mutation operations are applied to this set of parents, and children solutions are generated. These children solutions are stored in the set $Q_{t}$

3. A combined set $R_{t}=P_{t} \cup Q_{t}$ is formed. The fitness values of all the solutions of this set are calculated.

4. A non-dominated sorting of $R_{t}$ is done to obtain the solutions in various fronts.

5. A set $S_{t}$ is formed to keep the solutions chosen for the next generation. Until the size of $S_{t}$ is less than $N$, the solutions of the $1^{\text {st }}, 2^{\text {nd }}, 3^{\text {rd }}, \ldots, l^{\text {th }}$ fronts are selected.

6. If the size of $S_{t}$ is exactly equal to $N$, then $P_{t+1}=S_{t}$

7. Otherwise from the last front $\left(F_{l}\right), K=N-\bigcup_{i=1}^{l-1} F_{i}$ elements are selected. The elements of the last front are selected using association and niche preservation operators.

8. For association, first a set of reference-points is selected on a normalized hyper-plane. Using the axes intercepts of this plane, the fitness values of the solutions of the last front are normalized. Each solution-point is then assigned a reference-point, by measuring its distance from the line joining the origin with the reference-point and assigning it to the reference-point at the least distance.

9. For niche preservation, solution-points are chosen from the reference point having the least number of solution points associated with them. In case of more than one point being associated, the point with the least distance is chosen.
10. Once $K$ has reached its required value, $P_{t+1}$ is formed with the elements of $S_{t}$ up to $(l-1)$ th front and selected elements of the last front. The process is repeated for the required number of generations or up to convergence of solution.

\section{Results and observations}

In this section, the afore-mentioned model is solved using the Multi-objective Evolutionary Algorithm based on Decomposition and NSGA-III. Both the approaches are compared at the end of this section. In both cases, a small numerical example is illustrated.

\subsection{Multi-objective evolutionary algorithm based on decomposition (MOEA/D) approach}

In this section, MOEA/D by [35], with the Tchebycheff decomposition approach, has been used to solve the multiobjective problem. MOEA/D decomposes the multi-objective problem into $N$, in this paper taken as 100 , scalar optimization subproblems. These subproblems are solved simultaneously by evolving a population of solutions. A subproblem is associated with each individual solution in the population. Based on the distance of the weight vectors associated with each subproblem, a neighbourhood relation is developed. Optimization is done using the information from the neighbouring subproblem. The scalar optimization problem is of the form that minimizes $g^{t c}\left(x \mid \lambda^{j}, z\right)=\min _{1 \leq i \leq m} \frac{\lambda_{i}^{j} \mid\left(f_{i}-z_{i}\right)}{\left.\left(z^{n d d}\right)_{i}-z_{i}\right)} \mid$ subject to $x \in \Omega$ where $x$ is the chromosome, $m$ the number of objectives and $f_{i}$ is the fitness value of $i^{\text {th }}$ objective for the chromosome; $\lambda^{j}$ belongs to the set of even spread weight vectors $\lambda^{1}, \lambda^{2}, \ldots \lambda^{N}$; $z_{i}^{\text {nad }}$ is the largest value of $f_{i}$ in the current population and $\Omega$ the constraint space.

The initial size of population is taken as 100 and the number of generations is 200. A solution of this model is presented here.

For the given solution, cost $=\$ 209668.919$, deficiency in the supply chain is 57,073 and deficiency in the evacuation chain is 3767 . The chromosome of $Z_{j}$ for the solution is \begin{tabular}{l|l|l|l}
\hline & 0 & 3 & 0
\end{tabular} . The chromosome of $X_{i j}$ for the solution is \begin{tabular}{|l|l|l|l|l|l|l|l|l|}
\hline 0 & 1195 & 0 & 0 & 25193 & 0 & 0 & 996 & 0 \\
\hline
\end{tabular}

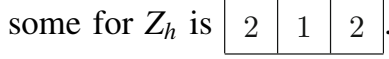

Table 4 contains the values of $\lambda_{j k m}, Y_{j k m}, \lambda_{h k p}$ and $N_{h k p}$ for the solution.

\subsection{NSGA-III approach}

In this section we have applied NSGA-III to solve the problem. The same data given in section 7 have been used. 
Table 4. $\lambda_{j k m}, Y_{j k m}, \lambda_{h k p}$ and $N_{h k p}$ for the solution.

\begin{tabular}{|c|c|c|c|c|c|c|c|}
\hline$j$ & $k=1$ & $k=2$ & $k=3$ & $h$ & $k=1$ & $k=2$ & $k=3$ \\
\hline 1 & $(0,0,0)$ & $(0,0,0)$ & $(0,0,0)$ & 1 & $(0,0,1)$ & $(0,1,0)$ & $(0,1,0)$ \\
\hline 2 & $(0,1,0)$ & $(1,0,0)$ & $(1,0,0)$ & 2 & $(0,1,0)$ & $(1,0,0)$ & $(0,0,1)$ \\
\hline 3 & $(0,0,0)$ & $(0,0,0)$ & $(0,0,0)$ & 3 & $(0,1,0)$ & $(0,1,0)$ & $(0,0,1)$ \\
\hline$j=1($ path $=1)$ & 0 & 0 & 0 & $h=1($ path $=1)$ & 0 & 0 & 0 \\
\hline$($ path $=2)$ & 0 & 0 & 0 & $($ path $=2)$ & 0 & 174 & 136 \\
\hline$($ path $=3)$ & 0 & 0 & 0 & $($ path $=3)$ & 380 & 0 & 0 \\
\hline$j=2($ path $=1)$ & 0 & 10859 & 4420 & $h=2($ path $=1)$ & 0 & 60 & 0 \\
\hline$($ path $=2)$ & 487 & 0 & 0 & $($ path $=2)$ & 1244 & 0 & 0 \\
\hline$($ path $=3)$ & 0 & 0 & 0 & $($ path $=3)$ & 0 & 0 & 863 \\
\hline$j=3($ path $=1)$ & 0 & 0 & 0 & $h=3($ path $=1)$ & 0 & 0 & 0 \\
\hline$($ path $=2)$ & 0 & 0 & 0 & $($ path $=2)$ & 12 & 293 & 0 \\
\hline$($ path $=3)$ & 0 & 0 & 0 & $($ path $=3)$ & 0 & 0 & 291 \\
\hline
\end{tabular}

Table 5. $\lambda_{j k m}, Y_{j k m}, \lambda_{h k p}$ and $N_{h k p}$ for the solution.

\begin{tabular}{|c|c|c|c|c|c|c|c|}
\hline$j$ & $k=1$ & $k=2$ & $k=3$ & $h$ & $k=1$ & $k=2$ & $k=3$ \\
\hline 1 & $(0,0,1)$ & $(1,0,0)$ & $(1,0,0)$ & 1 & $(0,0,1)$ & $(0,1,0)$ & $(0,0,1)$ \\
\hline 2 & $(0,0,0)$ & $(0,0,0)$ & $(0,0,0)$ & 2 & $(0,0,1)$ & $(1,0,0)$ & $(1,0,0)$ \\
\hline 3 & $(0,0,0)$ & $(0,0,0)$ & $(0,0,0)$ & 3 & $(1,0,0)$ & $(0,0,1)$ & $(0,1,0)$ \\
\hline$j=1($ path $=1)$ & 0 & 6542 & 16610 & $h=1($ path $=1)$ & 0 & 0 & 0 \\
\hline$($ path $=2)$ & 0 & 0 & 0 & $($ path $=2)$ & 0 & 465 & 0 \\
\hline$($ path $=3)$ & 10024 & 0 & 0 & $($ path $=3)$ & 650 & 0 & 91 \\
\hline$j=2($ path $=1)$ & 0 & 0 & 0 & $h=2($ path $=1)$ & 0 & 379 & 560 \\
\hline$($ path $=2)$ & 0 & 0 & 0 & $($ path $=2)$ & 0 & 0 & 0 \\
\hline$($ path $=3)$ & 0 & 0 & 0 & $($ path $=3)$ & 1768 & 0 & 0 \\
\hline$j=3($ path $=1)$ & 0 & 0 & 0 & $h=3($ path $=1)$ & 57 & 0 & 0 \\
\hline$($ path $=2)$ & 0 & 0 & 0 & $($ path $=2)$ & 0 & 0 & 527 \\
\hline$($ path $=3)$ & 0 & 0 & 0 & $($ path $=3)$ & 0 & 195 & 0 \\
\hline
\end{tabular}

The initial size of population is taken as 100 and the maximum number of generations is 1000 . The mutation probability has been taken as 0.1 . The purpose of applying NSGA-III to this problem is to provide a very wide range of options to the decision maker. The Pareto-optimal front will facilitate all such decisions. Presented here is one of the solutions of the first Pareto-optimal front.

For the given solution, cost $=\$ 312539.67$, deficiency in the supply chain is 39,663 and deficiency in evacuation chain is 2,528. Along the Pareto-optimal front, there will be relaxation in some objectives and improvement in others.

The chromosome of $Z_{j}$ is 1100.0 .

\begin{tabular}{c|c|c|c|c|c|c|c|c|c} 
The & \multicolumn{1}{c}{ chromosome } & \multicolumn{1}{c}{ of } & \multicolumn{1}{c}{$X_{i j}$} & is \\
\hline 17436 & 0 & 0 & 5299 & 0 & 0 & 13173 & 0 & 0 & . The chro-
\end{tabular}

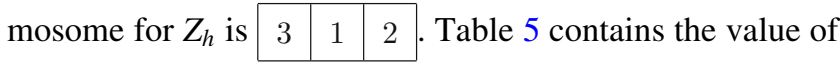
$\lambda_{j k m}, Y_{j k m}, \lambda_{h k p}$ and $N_{h k p}$ for the solution.

Pareto optimality is defined as a state of allocation of resources in which it is not possible to improve one solution without making the other solution worse. Figure 2 shows the Pareto-optimal front for the final generation.

The decision maker can choose a solution found by either MOEA/D or NSGA-III. Both the codes have been programmed on MATLAB on an Intel core ${ }^{t m}$ i5 processor. The

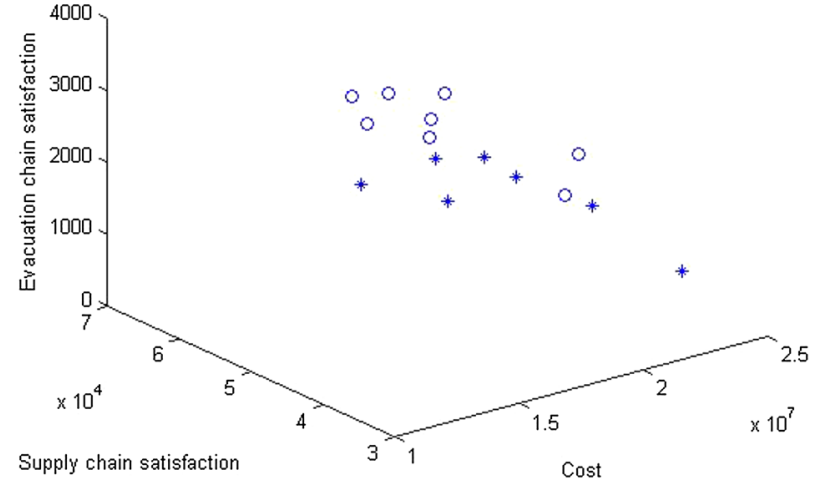

Figure 2. Pareto optimal front of final generation of MOEA/ D('o') and NSGA-III ('*').

run time for NSGA-III for 1000 generations was $3197 \mathrm{~s}$ and run time for MOEA/D for 200 generations was 4259 s.

\section{Conclusions and future scope}

In this paper, a multi-objective programming model has been proposed to optimize the evacuation operations and humanitarian relief in regard to the preparedness as well as the response phase. 
In an attempt to base our model as per the NDM guidelines, we have assumed that relief chain logistics constitute three stages and the evacuation chain logistics constitute two stages (see figure 1). The relief chain consists of three stages, i.e., the suppliers, the AA and the relief camps. The evacuation chain consists of two stages, i.e., the evacuation camp and AAs. The relief chain solution contains two decisions: first, the location of the relief camps and the transportation of goods from suppliers to relief camps and second, the amount of transportation from established relief camps to the AAs. For the evacuation chain, we have to decide on the location of the evacuation camps and the transportation of people from the AAs to the evacuation centres. Our multi-objective model minimizes three objectives. The first one is to minimize the set-up, procurement, transportation between supplier and relief camps, disruption penalty, transportation between relief camps and AAs, transportation between the AAs and evacuation camps, risk penalty cost and stay cost at evacuation camp. The second and third objectives, minimize the gap between demand and supply of the relief and evacuation chains, respectively. We have taken more than one path between any camp and the affected areas. This makes our model more resilient in terms of the distribution of goods from relief camps to the AAs. In the post-disaster scenario, if all the three paths have been disrupted, then we can use helicopters or boats for the transportation.

We solved our model using two approaches, MOEA/D and NSGA-III. In the first approach, neighbourhood relations are developed between the subproblems, and optimization is done only by using the information from the neighbourhood. The main objective of the second approach is to find the set of non-dominating solutions. In this approach, the decision maker has many options. This model is solved through an evolutionary algorithm called NSGAIII. We cannot relax objective 2 and 3, i.e. customer satisfaction, to a large extent; hence only the 1st non-dominated front has been presented in the Results section. MATLAB was used for the programming of NSGA-III.

There is ample scope for development of the approach in this paper. Since NSGA-III can be used to solve problems of up to 15 objectives, we can include more objectives like taking risks (disruption of road network) as another objective instead of including it in the cost. We have not considered the problem of vehicle scheduling but the constraints due to the limitation of man power and types of vehicle available should also be considered in such disaster relief logistics models. The demands in the AAs can be taken as stochastic rather than deterministic to make the model more realistic and practical.

In order to check the performance of this model in a real life scenario, a case study of some disaster-prone areas can be carried out. The flood-prone area of Bihar and Mumbai can serve as good examples for the application of this model. The model can be made more realistic by making the supply and evacuation chain dynamic, i.e., it can incorporate any newly requested demand for emergency supplies and evacuation. Bayesian decision theory can be used to forecast the demand in different AAs, based on the characteristics of the area and the historical data.

\section{References}

[1] NDMA I National Disaster Management Guidelines (NDMA) I January 2008, Management of floods. National Disaster Management Authority, Government of India, http:// www.ndma.gov.in/images/guidelines/flood.pdf

[2] NIDM I. June 2012 India disaster report, 2011. National Institute of Disaster Management, Government of India, http://nidm.gov.in/PDF/pubs/India

[3] Biswas T and Samanta S 2016 A strategic decision support system for logistics and supply chain network design. Sadhana 41(6): 583-588

[4] Jauhar S K and Pant M 2016 Genetic algorithms in supply chain management: a critical analysis of the literature. Sadhana 41(9): 993-1017

[5] Kovacs G and Spens K M 2007 Humanitarian logistics in disaster relief operations. Int. J. Phys. Distrib. Logist. Manage. 37(2): 99-114

[6] Van Wassenhove L N 2006 Humanitarian aid logistics: supply chain management in high gear. J. Oper. Res. Soc. 57(5): 475-489

[7] Altay N and Green III W G 2006 OR/MS research in disaster operations management. Eur. J. Oper. Res. 175(1): 475-493

[8] John L and Ramesh A 2012 Humanitarian supply chain management in india: a sap-lap framework. J. Adv. Manage. Res. 9(2): 217-235

[9] Kabra G and Ramesh A 2015 An empirical investigation of the enablers in humanitarian supply chain management in india: a case study. J. Adv. Manage. Res. 12(1): 30-42

[10] Toregas C, Swain R, ReVelle C and Bergman L 1971 The location of emergency service facilities. Oper. Res. 19(6):1363-1373

[11] Parentela E M and Nambisan S S 2000 Emergency response (disaster management). In: Urban planning and development applications of GIS. Reston, VA: ASCE, pp. 181-196

[12] Akkihal A R 2006 Inventory pre-positioning for humanitarian operations. Master's thesis, Massachusetts Institute of Technology

[13] Balcik B and Beamon B M 2008 Facility location in humanitarian relief. Int. J. Logist. Res. Appl. 11(2): 101-121

[14] Tzeng G H, Cheng H J and Huang T D 2007 Multi-objective optimal planning for designing relief delivery systems. Transport. Res. Part E: Logist. Transport. Rev. 43(6): 673-686

[15] Ukkusuri S and Yushimito W 2008 Location routing approach for the humanitarian prepositioning problem. Transport. Res. Rec. J. Transport. Res. Board 2089: 18-25

[16] Ozdamar L, Ekinci E and Kucukyazici B 2004 Emergency logistics planning in natural disasters. Ann. Oper. Res. 129(1-4): 217-245

[17] Caunhye A M, Nie X and Pokharel S 2012 Optimization models in emergency logistics: a literature review. SocioEcon. Plann. Sci. 46(1): 4-13 
[18] Whybark D C 2007 Issues in managing disaster relief inventories. Int. J. Prod. Econ. 108(1-2): 228-235

[19] Ozbay K and Ozguven E E 2007 Stochastic humanitarian inventory control model for disaster planning. Transport. Res. Rec. J. Transport. Res. Board 2022: 63-75

[20] Beamon B M and Kotleba S A 2006 Inventory modelling for complex emergencies in humanitarian relief operations. Int. J. Logist. Res. Appl. 9(1): 1-18

[21] Rachaniotis N P, Dasaklis T, Pappis C P and van Wassenhove L N 2013 Multiple location and routing models in humanitarian logistics. In: Zeimpekis V, Ichoua $\mathrm{S}$ and Minis I (Eds.) Humanitarian and relief logistics, vol. 54, Operations Research/Computer Science Interfaces Series. New York: Springer, pp. 43-57

[22] Saadatsereshta M, Mansourianb A and Taleai M 2009 Evacuation planning using multi-objective evolutionary optimization approach. Eur. J. Oper. Res. 198(1): 305-314

[23] Stepanov A and Smith J M 2009 Multi-objective evacuation routing in transportation networks. Eur. J. Oper. Res. 198(2): 435-446

[24] Yi W and Kumar A 2007 Ant colony optimization for disaster relief operations. Transport. Res. Part E: Logist. Transport. Rev. 43(6): 660-672

[25] Yi W and Ozdamar L 2007 A dynamic logistics coordination model for evacuation and support in disaster response activities. Eur. J. Oper. Res. 179(3): 1177-1193

[26] Regnier E 2008 Public evacuation decisions and hurricane track uncertainty. Manage. Sci. 54(1): 16-28

[27] Zhou Q, Huang W and Zhang Y 2011 Identifying critical success factors in emergency management using a fuzzy DEMATEL method. Saf. Sci. 49(2): 243-252
[28] Ben-Tal A, Chung B D, Mandala S R and Yao T 2011 Robust optimization for emergency logistics planning: risk mitigation in humanitarian relief supply chains. Transp. Res. Part B: Methodol. 45(8): 1177-1189

[29] de la Torre L E, Dolinskaya I S and Smilowitz K R 2012 Disaster relief routing: integrating research and practice. Socio-Econ. Plann. Sci. 46(1): 88-97

[30] Sheu J B 2007 An emergency logistics distribution approach for quick response to urgent relief demand in disasters. Transport. Res. Part E: Logist. Transport. Rev. 43(6): 687-709

[31] Sheu J B 2010 Dynamic relief-demand management for emergency logistics operations under large-scale disasters. Transport. Res. Part E: Logist. Transport. Rev. 46(1): 1-17

[32] Lodree Jr E J and Taskin S 2008 An insurance risk management framework for disaster relief and supply chain disruption inventory planning. J. Oper. Res. Soc. 59(4): 674-684

[33] Hadiguna R A, Kamil I, Delati A and Reed R 2014 Implementing a web-based decision support system for disaster logistics: a case study of an evacuation location assessment for Indonesia. Int. J. Disaster Risk Reduct. 9: 38-47

[34] Jain H and Deb K 2013 An improved adaptive approach for elitist nondominated sorting genetic algorithm for manyobjective optimization. In: Purshouse R, Fleming P, Fonseca C, Greco S and Shaw J (Eds.) Evolutionary multi-criterion optimization, vol. 7811, Lecture Notes in Computer Science. Berlin-Heidelberg: Springer

[35] Zhang Q and Li H 2007 MOEA/D: a multiobjective evolutionary algorithm based on decomposition. IEEE Trans. Evol. Comput. 11(6):712-731 